\title{
Multimodale Therapie von Lebermetastasen
}

\section{Multimodal Treatment of Liver Metastases}

\author{
Gesprächsleiter: E. Klar, Rostock M. Birth, Lübeck \\ Teilnehmer: \\ W.O. Bechstein, Frankfurt/M. \\ R.W. Günther/J. Tacke, Aachen \\ T. Helmberger, München \\ M. Freund, Rostock
}

\section{Frage 1: Welche Art der Bildgebung halten Sie für ent- scheidend in der Differenzierung von Raumforderungen der Leber?}

Bechstein: Bei den meisten Patienten wird eine intrahepatische Raumforderung zunächst durch Sonographie erfasst. Typische Befunde wie Leberzysten, Metastasen sowie hepatozelluläre Karzinome (HCCs) in Zirrhose und Hämangiome können gut durch Sonographie dargestellt werden. Ein Schichtbildverfahren erscheint, außer im Fall von sonographisch typischen Hämangiomen und Leberzysten, dennoch wünschenswert und hier am besten ein DünnschichtspiralCT mit Kontrastmittel-Verstärkung. Besonders beim HCC in Zirrhose hat sich die Kontrastmittel-gestützte MRT bewährt.

Günther/Tacke: Für alle bildgebenden Verfahren gilt mehr oder weniger die Faustregel, dass benigne Raumforderungen der Leber gut und maligne schlechter zu differenzieren sind. Die Sonographie ist aufgrund der hohen Verfügbarkeit, der akzeptablen Aussagekraft und kostengünstigen Leistung das primär am häufigsten eingesetzte Verfahren bei der Suche und Differenzierung von Raumforderungen der Leber. Zysten und typische Hämangiome sind damit in der Regel gut zu differenzieren. Auch eine Differenzierung von Leberadenom und fokal-nodulärer Hyperplasie ist möglich, erfordert aber häufig eine Bestätigung durch die CT. Die früher bei dieser Fragestellung durchgeführte Szintigraphie ist durch CT und MRT ersetzt. Eine nähere sonomorphologische Einordnung ist selbst bei größeren solitären Lebermalignomen schwierig, in Kombination mit klinischen Information (Grunderkrankung) und Laborwerten (z.B. Alpha-Fetoprotein) meist jedoch möglich. Auch bei den anderen bildgebenden Verfahren ist letzteres wichtig. Bei bestehender Leberzirrhose, Fettleber oder anatomisch ungünstigem Schallfenster sind die sonogra- phischen Möglichkeiten von Tumornachweis und -differenzierung zusätzlich erschwert.

Das Standardverfahren zum Nachweis und Klassifizierung von Leberraumforderungen ist die 3-Phasen-Spiral-CT - am besten die Multidetektorspiral-CT. Noch etwas empfindlicher scheint die Hochfeld-MRT zu sein, sie ist jedoch mit höheren Kosten verbunden.

Mit der CT wie mit der MRT lassen sich durch i.v. Kontrastmittel-Gabe aufgrund von nativen Dichtewerten in der CT und aufgrund der Signalintensität in der MRT sowie zeitlichem Anreicherungsverhalten bestimmte Raumforderungen näher differenzieren. Zysten, Hämangiome, Lipome, fokalnoduläre Hyperplasie und Angiomyolipome sind in der Regel gut einzuordnen. Schwieriger wird es beim Leberadenom. Die Differenzierung von malignen primären Lebertumoren (primäres Leberzellkarzinom, cholangioläres Karzinom) kann problematisch sein, zumal das HCC ein breites morphologisches Erscheinungsbild bietet, das von stark hypervaskulär bis hypovaskulär sowie von umschrieben bis multifokal und diffus wachsend reichen kann. Dementsprechend schwierig kann die Einordnung sein. Die Differenzierung von dysplastischen Herden und kleinen HCCs bei Leberzirrhose stellt eine besondere Herausforderung dar, die nicht zufriedenstellend zu lösen ist. Die Einordnung von multiplen Lebermetastasen ist - abgesehen von der generellen Einschränkung der Erkennbarkeit erst ab einer Mindestgröße von zirka $10 \mathrm{~mm}$ - insbesondere auch in Zusammenhang mit der Grunderkrankung möglich.

Für den Nachweis und die Differenzierung von Leberraumforderungen steht die CT an erster Stelle in der Diagnostik. In unklaren Fällen und bei Kontraindikationen gegen jodhaltige Röntgenkontrastmittel ist die MRT angezeigt. In seltenen Fällen kann bei der Suche und Differenzierung von Lebermetastasen auch die diesbezüglich sehr sensitive FDG-PETUntersuchung weiterführen.

\begin{tabular}{ll}
\hline KARGER & ( ) 2003 S. Karger GmbH, Freiburg \\
Fax +49 761 4520714 & Accessible online at: \\
$\begin{array}{l}\text { E-mail Information@Karger.de } \\
\text { www.karger.com }\end{array}$ & www.karger.com/cga
\end{tabular}

Prof. Dr. med. Ernst Klar

Abteilung für Allgemein-, Thorax-, Gefäß- und Transplantationschirurgie

Chirurgische Klinik und Poliklinik, Universität Rostock

Schillingallee 35, D-18057 Rostock

Tel. +49 381494 60-01, Fax -02

E-mail Ernst.Klar@med.uni-rostock.de 
Freund: Standard ist sicherlich das Spiral-CT. Es stehen aber auch moderne sonographische Verfahren zur Verfügung, die z.B. eine sichere Differentialdiagnose von Hämangiomen erlauben. Sonographische Verfahren sind stark von den lokalen Gegebenheiten und der Erfahrung des Untersuchers abhängig. In der Differenzierung neuroendokriner Tumoren sollten die spezifischen nuklearmedizinischen Untersuchungsverfahren wie ( ${ }^{111} \mathrm{In}$ )-DTPAOC (Octreoscan ${ }^{\circledR}$ zur Detektion des Somatostatin-Rezeptors nicht vergessen werden.

\section{Frage 2: Welche Techniken der Bildgebung besitzen für Sie die höchste Sensitivität beim Nachweis von Leber- metastasen?}

Bechstein: Das Dünnschichtspiral-CT mit Kontrastmittel-Verstärkung besitzt nach meinem persönlichen Eindruck die höchste Sensitivität für die Detektion von Lebermetastasen. Bei unklaren Befunden kann ergänzend eine MRT durchgeführt werden.

Günther/Tacke: Mit bildgebenden Verfahren sind primäre und sekundäre maligne Lebertumoren aber einer Größe von zirka 5-10 mm nachzuweisen.

Nach einer Meta-Analyse von Kinkel et al. [1] hat in den Studien, deren Spezifität höher als $85 \%$ war, die transabdominelle Sonographie beim Nachweis von Lebermetastasen eine Sensitivität von $55 \%$, die CT von $72 \%$ und die MRT von $76 \%$ und die FDG-PET von 90\%. Die intraoperative Sonographie hat eine hohe Sensitivität und Spezifität beim Nachweis von Lebermetastasen.

Für die Objektivierung des Therapieerfolgs im Verlauf einer Behandlung von Lebermetastasen ist die perkutane Sonographie weniger geeignet als CT und MRT.

Im Hinblick auf die akzeptable Sensitivität und Spezifität, die Verfügbarkeit sowie die Kosten bleibt in praxi für die Routinediagnostik die CT das wichtigste Verfahren beim Nachweis von Lebermetastasen, wenngleich die MRT den Vorteil der höheren Sensitivität, der fehlenden Strahlenbelastung und der höheren Nachweisrate von kleinen Lebermetastasen bei Anwendung leberspezifischer Kontrastmittel bietet.

Freund: Standard ist auch hier das Spiral-CT, während das MRT noch unterlegen ist. Sensible sonographische Untersuchungen können bereits präoperativ eine bessere Sensitivität ergeben [2]. Hervorragende Ergebnisse liefert die intraoperative Ultraschalluntersuchung [3].

\section{Frage 3: Besitzt die Chemoembolisation einen Stellen- wert in der Therapie von Lebermetastasen?}

Bechstein: Insbesondere bei Lebermetastasen neuroendokriner Tumoren kann die Chemoembolisation nützlich sein, bei kolorektalen Lebermetastasen kommt sie nur ausnahmsweise zur Anwendung. Auch Metastasen des C-Zell-Karzinoms (medulläres Schilddrüsenkarzinom) können durch Chemoembolisation behandelt werden.

Günther/Tacke: Die Überlebenszeit von Patienten mit Lebermetastasen unterschiedlicher Primärtumoren wird durch eine Chemoembolisation nicht signifikant verbessert. Das Verfahren ist daher für diesen Zweck nicht sinnvoll einzusetzen.

Helmberger: Die klassische Chemoembolisation besitzt keinen Stellenwert, da die «üblichen» Lebermetastasen für diese Therapie nicht sensitiv sind. Die teilweise berichteten positiven Daten sind durch Studien nicht untermauert. Eine Ausnahme stellen hypervaskularisierte Metastasen, so z.B. von Nierenzellkarzinomen oder neuroendokrinen Tumoren, dar, die durch eine transarterielle Embolisation behandelt werden können. Auch hier hat die Beimengung eines Chemotherapeutikums keinen Sinn.

Freund: Die Chemoembolisation ist eine palliative Therapiemaßnahme. Sie sollte nicht bei resektablen Lebermetastasen eingesetzt werden. Sie wird von einigen Untersuchern mit anderen lokalen Therapieverfahren z.B. der Thermoablation kombiniert. Für einen sinnvollen palliativen Einsatz ist darüber hinaus Voraussetzung, dass die Lebermetastasierung für den Patienten klinisch die zentrale Bedeutung hat. Das Verfahren spielt bei metastasierten Karzinoid oder auch beim hepatozellulären Karzinom eine Rolle.

\section{Frage 4: In welchen Fällen unterstützen Sie eine neoad- juvante Chemotherapie vor geplanter Operation?}

Bechstein: Eine große multizentrische Studie zur Klärung des Stellenwerts der neoadjuvanten Chemotherapie bei resektablen Lebertumoren befindet sich derzeit noch in der Endphase der Rekrutierung (EORTC 40983), so dass der Stellenwert der neoadjuvanten Chemotherapie bei resektablen Metastasen bisher nicht geklärt ist. Es gibt jedoch eine Reihe von Veröffentlichungen, die zeigen können, dass durch eine präoperative Chemotherapie insbesondere unter Verwendung von Oxaliplatin die Resektionsraten gesteigert werden können und zuvor nicht resektabel erscheinende Metastasen dann doch einer R0-Resektion zugeführt werden können $[4,5]$. Dies betrifft nach eigener Erfahrung insbesondere große und anatomisch ungünstig gelegene Metastasen (in der Nähe der Mündung der drei großen Lebervenen in die Vena cava bzw. im Bereich der Pfortaderaufzweigung im Leberhilus).

Freund: Zwei Studiengruppen haben sich dieser Fragestellung in prospektiven Studien bei kolorektalen Karzinomen gewidmet [6-8]. Patienten mit primär nichtresektablen Le- 
bermetastasen wurden intravenös mit 5-Fluorouracil, Folinsäure und Oxaliplatin behandelt. Zwischen 13,5 und 38\% der Patienten konnten im Anschluss an die Chemotherapie komplett reseziert werden. Das Langzeitüberleben lag für die Patienten mit Resektion bei 40-50\%. Die Berichte beziehen sich auf mehr als 200 Patienten und stehen damit auf einer soliden Basis. Dennoch darf nicht übersehen werden, dass auch Selektionseffekte eine Rolle spielen können. Insgesamt sollte man in der Routinesituation pragmatisch vorgehen: Bei primär aufgrund der Größe, Lage, Anzahl oder in speziellen Fällen auch zusätzlicher extrahepatischer Manifestation nichtresektablen Lebermetastasen bei kolorektalen Tumoren sollte eine wirksame Kombinations-Chemotherapie eingeleitet werden. Es sollte wie bei jeder Therapie regelmäßige Evaluationen durchgeführt werden. Bei ihnen ist dann auch zu prüfen, ob nicht doch auch eine Resektabilität erreicht wird. Schließlich möchte ich für die Teilnahme an der EORTC-Studie 40983 werben. Die Studie prüft randomisiert die Frage einer neodajuvanten plus adjuvanten Chemotherapie mit Oxaliplatin und Fluorouracil/Folinsäure versus einer alleinigen Resektion. Auf ihrer Grundlage wird die Frage der neoadjuvanten Therapie hoffentlich bald abschließend beurteilt werden können [9].

Noch ein Wort zu anderen Tumoren: In vielen anderen Situationen wird eine Resektion aus Gründen der systemischen Aussaat der Erkrankung (Beispiel Mammakarzinom), oder aus Gründen der Biologie der Erkrankung (Bronchialkarzinom) nicht sinnvoll sein. Bei anderen Erkrankungen wie dem Nierenzellkarzinom stehen uns keine ausreichend wirksamen Therapien zur Verfügung, ist also eine neodajuvante Therapie nicht sinnvoll oder möglich.

Im weiteren Sinne mag man Hodenkarzinome in diesem $\mathrm{Zu}-$ sammenhang sehen. Hier ist nach Chemotherapie öfter die Notwendigkeit zur Resektion von Teratomen, gelegentlich auch in der Leber, gegeben.

\section{Frage 5: Ist für Sie eine adjuvante Chemotherapie nach RO-Resektion kolorektaler Lebermetastasen sinnvoll?}

Bechstein: Bisher gibt es keine Studiendaten, die den Stellenwert einer adjuvanten Chemotherapie nach R0-Resektion kolorektaler Lebermetastasen belegt haben. Eine entsprechende multizentrische Studie zur adjuvanten Therapie nach R0-Resektion unter Verwendung von Irinotecan befindet sich derzeit in der Rekrutierungsphase. Allerdings verwenden wir in Einzelfällen eine adjuvante Chemotherapie nach R0-Resektion kolorektaler Lebermetastasen, wenn diese synchron mit der Erstdiagnose eines kolorektalen Karzinoms aufgetreten sind, welches zuvor oder gleichzeitig entfernt wurde (jeweils Dukes-D-Stadium, UICC-Stadium IV). Diesen Patienten keine adjuvante Chemotherapie zu geben, würde bedeuten, eine suboptimale Therapie durchzuführen, wenn man nur den Lokalbefund des Kolonkarzinoms in Betracht zieht. In den meisten Fällen handelt es sich ja hier um nodal positives Stadium, so dass eine adjuvante Chemotherapie sinnvoll erscheint.

Freund: In der adjuvanten Situation sind nach R0-Resektion in verschiedenen Studien vor allem lokale Chemotherapieansätze, teils auch in Kombination mit systemischer Chemotherapie verfolgt worden [10-12]. Aus den Studien lässt sich keine verlässliche Evidenz für die Wirksamkeit einer adjuvanten Therapie in dieser Situation ableiten. Allerdings muss darauf hingewiesen werden, dass die Daten aus der Ära vor den Kombinationstherapien stammen. Unter den Möglichkeiten, die wir heute mit der Kombination mit Oxaliplatin und Irinotecan haben, vielleicht auch vor dem Hintergrund neuer Entwicklungen wie z.B. dem EGFR-Antikörper Cetuximab müsste diese Frage sicherlich erneut untersucht werden. Sie ist zum Teil auch in der erwähnten EORTC-Studie 40983 enthalten [9].

\section{Frage 6: Unterstützen Sie die Anwendung lokaler Chemotherapie?}

Bechstein: Die regionale Chemotherapie von Lebermetastasen erscheint aufgrund jüngster multizentrischer Daten [13] überholt, obwohl bei isolierter hepatischer Metastasierung ein gutes Ansprechen beobachtet werden kann.

\section{Günther/Tacke: Nein.}

Freund: Eine hochrangig publizierte randomisierte Studie zeigt die Überlegenheit einer lokalen Chemotherapie in Kombination mit der systemischen in der adjuvanten Situation nach R0-Resektion bei kolorektalen Tumoren [12]. Dennoch wird die lokale Therapie de facto nur von wenigen Untersuchern verfolgt. Dies mag daran liegen, dass nur wenige Zytostatika wie FUDR wirklich einen für eine lokale Therapie ausreichenden «First-pass»-Effekt aufweisen. Außerdem sind die Komplikationsraten bei lokaler Chemotherapie mit den notwendigen Kathetersystemen nicht zu vernachlässigen [14]. Insgesamt bleibt eine lokale Chemotherapie besonderen Situationen und Institutionen vorbehalten. Eine Verbreiterung der Datenlage zum Nutzen dieser Therapiemodalität in vergleichenden Studien wäre sehr wünschenswert.

\section{Frage 7: Gibt es ein Verfahren der lokalen Thermoabla- tion, das mit kurativer Zielsetzung eingesetzt werden darf?}

Bechstein: Es gibt zahlreiche Daten, die die Vermutung nahe legen, dass insbesondere die laserinduzierte Thermotherapie zu einer kontrollierten, ausreichenden Nekroseinduktion von Lebermetastasen führt. Allerdings gibt es bisher keine rando- 
misierte Studie, die dieses Verfahren «head-to-head» mit der Leberresektion vergleicht. Insbesondere ist in allen Studien auch immer wieder erschreckend, dass sich, trotz optimaler Bildgebung, beim offenen Vorgehen, sei es für Leberteilresektionen, sei es für lokal ablative Therapie, in einem relativ hohen Prozentsatz zusätzliche Befunde und mitunter auch extrahepatische Manifestationen finden, die der präoperativen Bildgebung nicht zugänglich waren.

Günther/Tacke: Nach den Gesichtspunkten evidenzbasierter Medizin gibt es kein solches Verfahren, da kein thermisches Ablationsverfahren in einer prospektiv-randomisierten Studie gegen die chirurgische Resektionsverfahren untersucht wurde und alle bisherigen Bemühungen daran scheiterten, die Gruppe der zu operierenden Patienten zu akquirieren. Allerdings gilt sowohl für die Laserablation als auch für die Radiofrequenzablation, dass nach den bisherigen Literaturergebnissen diese Verfahren bei Patienten mit Lebermetastasen kolorektaler Karzinome gleich gute Überlebenszeiten wie die operative Resektion aufweisen. Wenn zudem berücksichtigt wird, dass bei den Kriterien der R0-Resektion $80 \%$ aller Patienten innerhalb von 5 Jahren ein Rezidiv erleben, kann auch von keiner «kurativen» Resektion gesprochen werden.

Helmberger: Meines Erachtens kann system- bzw. methodenbedingt derzeit keines der lokal ablativen Verfahren primär zur kurativen Therapie eingesetzt werden:

1) Eine direkte histopathologische Kontrolle des Ablationsergebnisses, wie es bei einer chirurgischen Resektion im Sinne von R0-2 möglich ist, ist methodenbedingt bei den thermoablativen Verfahren nicht möglich, d.h., ob sich um eine «T0-Thermoablation» gehandelt hat, kann nur über den Verlauf beurteilt werden. Das schließt allerdings nicht aus, das das Verfahren mit kurativer Intention eingesetzt wird. Da jedoch auch für den primär kurativen Einsatz keine Studien vorliegen, ist diese Intention vermutlich nicht ganz lege artis.

2) Bei den meisten Patienten, die in den Zentren behandelt, werden, handelt es sich um Patienten, bei denen das Leiden bereits sehr fortgeschritten ist, so dass sich die Frage nach einer Kuration nicht stellt.

Eine Ausnahme könnte auch hier die direkte, z.B. intraoperative, Thermoablation darstellen, bei der das Ablationsergebnis zumindest mit einer Art Sichtprüfung auf Vollständigkeit untersucht werden kann und das Thermoablationssystem als erweitertes chirurgisches Instrumentarium betrachtet wird.

Freund: Die lokale Thermoablation mit Anwendung von Laser- oder Radiofrequenzablation ist grundsätzlich ein palliatives Therapieverfahren. Ist eine kurative Zielsetzung gegeben, sollte eine Resektion bevorzugt werden. Sie hat eine gut belegte kurative Potenz [15-17], während verlässliche Daten für die Thermoablation ausstehen.

\section{Frage 8: Bewerten Sie die lokal ablativen Verfahren hin- sichtlich der Applikation perkutan oder intraoperativ.}

Bechstein: Der technische Aufwand der laserinduzierten Thermotherapie mit Online-MRT-Kontrolle macht ein offenes Therapieverfahren nicht möglich. Die Entscheidung zwischen perkutaner und intraoperativer Therapie bleibt somit letztlich für die Radiofrequenzablation bzw. Kryotherapie. Auch hierbei gilt, dass beim offenen Verfahren zusätzliche Befunde zuverlässiger entdeckt werden können als beim perkutanen Verfahren. Dies betrifft nicht nur mögliche extrahepatische Manifestationen des Tumors, sondern auch zusätzliche intrahepatische Läsionen. Ein weiterer Vorteil des offenen Vorgehens besteht in der Möglichkeit der temporären Okklusion des Ligamentum hepatoduodenale (Pringle-Manöver). Hierdurch können insbesondere Metastasen in der Nähe von größeren Gefäßen sicherer komplett abladiert werden.

Günther/Tacke: Grundsätzlich sind beide Applikationsarten möglich und werden auch in großen Studien mit gutem Erfolg eingesetzt. Die intraoperative Thermoablation hat ihre Indikation in der zusätzlichen Ablation von Herden, die nicht resektiv angegangen werden können und in der gleichen Sitzung wie resektable Metastasen behandelt werden. Allerdings werden diese ausschließlich sonographisch kontrolliert abladiert, was eine entsprechende Erfahrung mit der Methode voraussetzt. Eine Laparatomie zum ausschließlichen Zweck einer Thermoablation ist derzeit kaum indiziert, da die Ablation in den meisten Fällen auch perkutan erfolgen kann. Eine Ausnahme können Tumoren sein, zu deren vollständiger Ablation die Dissektion verletzlicher Strukturen (wie Magen oder Gallenblase) notwendig ist. Die Mehrzahl aller Tumoren kann, sofern die Kriterien einer vollständigen Ablation gegeben sind, perkutan behandelt werden.

\section{Helmberger:}

- Dei Radiofrequenzablation ist geeignet für perkutanen und intaoperativen Einsatz.

- Die Laserablation ist geeignet für perkutanen und intraoperativen Einsatz. Die Systeme, die mit Hüllkatheter arbeiten, sind allerdings relativ dicklumig (zirka $3 \mathrm{~mm}$ ), so dass bei perkutaner Anwendung in der Regel der Zugangsweg verklebt werden muss.

- Die Kryotherapie ist derzeit nur intraoperativ verfügbar, es gibt kein System mehr für den perkutanen Einsatz.

Radiofrequenzablation und Laserablation sind grundsätzlich intraoperativ anwendbar, problematisch ist lediglich das Monitoring per Ultraschall, bei dem es sich um die schwächste Methode zum Monitoring handelt. Zieht man also die Möglichkeiten der Bildgebung mit in Betracht, so ist einer sicheren Sonden- und Läsionsidentifizierung, z.B. mit der CT, der Vorzug zu geben. Insofern dürfte der schwerwiegendste und für den Outcome entscheidende Unterschied im Monitoring liegen. Andererseits sind bestimmte, die Ablation verstärken- 
de Manöver (z.B. Pringle) nur offen operativ sicher durchführbar.

Freund: Es existieren keine vergleichenden Studien, sondern nur mehr oder weniger umfangreiche meist retrospektive Auswertungen [18-22]. Naturgemäß wird eine perkutane lokale Ablation bei Lebermetastasen vor allem im palliativen Sinne eingesetzt. Einige Autoren weisen auf eine verbesserte Resektionsrate bei primären oder sekundären Lebertumoren nach intraoperativer Radiofrequenzablation. Es bleibt abzuwarten, ob ein Vorteil in vergleichenden Untersuchungen gezeigt werden kann.

\section{Frage 9: Welche Tumorcharakteristika limitieren die lokale Thermoablation?}

Bechstein: Zunächst einmal die Größe der Tumoren, Tumoren $>4 \mathrm{~cm}$ erscheinen für die lokale Thermoablation nicht geeignet. Zweitens auch die Lage der Tumoren, insbesondere wenn diese sehr nahe an großen Gefäßen, seien es Pfortaderäste oder Lebervenen, liegen, ist die Nekroseinduktion in Gefäßnähe häufig nicht hinreichend.

Günther/Tacke: Da bei der Thermoablation grundsätzlich einer der chirurgischen R0-Resektion entsprechende Situation angestrebt wird (d.h. Ablation im Gesunden mit entsprechendem Sicherheitssaum), stellt eine exzentrische Lage eines Tumors am Rand der Leber ein Problem dar. So kann beispielsweise ein breitbasig dem Zwerchfell aufsitzender Tumor naturgemäß nicht im Gesunden abladiert werden. Ebenso verhält es sich mit Läsionen im Lebersegment 2, wenn diese Kontakt sowohl zum Zwerchfell als auch zum Magen haben. Eine zentral-portale Lage mit Tumorkontakt zum Ductus choledochus stellt ebenfalls eine Kontraindikation zur Thermoablation dar. Eine weitere Einschränkung ist die Größe der einzelnen Läsionen. Derzeit können zwar Tumorgrößen bis $7 \mathrm{~cm}$ behandelt werden, jedoch stellt dies nur Einzelfälle dar, da mehrere Läsionen dieser Größe bei einem Patienten nicht erfolgreich im Gesunden behandelbar sind. Daher liegt die maximale Größe bei mehreren Herden derzeit bei 3,5-4 cm.

Helmberger: In unserer Erfahrung sind die Hauptlimitationen eine Tumorgröße $>5 \mathrm{~cm}$, die Lage und die Gefäßnähe. Mit der entsprechenden Bildgebung und Guidance-Systemen kann jede Region der Leber perkutan erreicht werden.

Alle Verfahren, ob perkutan oder offen angewendet, tendieren zur «Unterablation»!

Freund: Im Prinzip stellt sich die Frage ähnlich wie für die Resektion: Eine Thermoablation ist bei systemischer Dissemination sowie bei ausgedehnten und zahlreichen und bei synchronen Lebermetastasen nicht sinnvoll.
Frage 10: Nichtkolorektale Lebermetastasen: Bei welcher Art halten Sie die chirurgische Therapie für kontraindiziert? Bei welchem Typ ist ein operatives Vorgehen vertretbar trotz uneinheitlicher Datenlage?

Bechstein: Zunächst einmal ist die Möglichkeit zur Leberresektion beschränkt durch Lokalisation und Zahl der Metastasen. Bei solitären metachronen Metastasen ist die Datenlage sehr uneinheitlich, und hier kann ich keine klaren Kontraindikationen erkennen. Bei multilokulären Metastasen ist dies sehr viel schwieriger zu beantworten. Letztlich spielt bei der individuellen Entscheidung auch immer die Gesamtsituation des Patienten einschließlich Lebensalter und der Möglichkeit verfügbarer anderer Therapien (Chemotherapie) eine Rolle, wobei die radikale Operation bei Erzielung einer R0-Resektion eine formale Vollremission ermöglichen kann.

Freund: Eine lokale Komponente wie die Resektion von Lebermetastasen ist nur bei entsprechender Biologie des Tumors sinnvoll in ein Therapiekonzept einzubauen. Das Mammakarzinom ist in der Regel ein disseminierter Tumor. Zudem sind Lebermetastasen ein Zeichen für einen prognostisch ungünstigen Verlauf. Im Gegensatz zu einigen optimistischen Berichten kann eine Resektion generell nicht empfohlen werden [25, 26].

Die Resektion von Lebermetastasen bei neuroendokrinen Tumoren ist bei Operabilität sinnvoll mit guten 5-JahresÜberlebensraten [27, 28]. Allerdings erleiden im weiteren Verlauf dann doch die meisten Patienten ein Rezidiv [29].

Verbleibende Teratome nach erfolgreicher Chemotherapie eines Hodenkarzinoms bilden eine besondere Situation. Eine Resektion ist indiziert, wenn möglich. Eine Indikation zur Operation von Lebermetastasen kann sich bei Patienten mit Nierenzellkarzinom sowie bei einzelnen Patienten mit Melanomen und Sarkomen ergeben [26].

\section{Frage 11: Würden Sie die chirurgische Resektion und Thermoablation kombinieren? Wann würden Sie die Thermoablation intraoperativ, wann postoperativ anwenden?}

Bechstein: Die kombinierte Anwendung von Leberteilresektion und Thermoablation kommt insbesondere dann in Frage, wenn zum einen eine größere Leberteilresektion von mehr als drei Segmenten erforderlich wird und sich zum anderen im kontralateralen Leberlappen eine zentrale Läsion befindet. In diesen Fällen führen wir eine kombinierte Therapie durch. Eine postoperative Therapie erscheint wenig sinnvoll, außer im Fall von neu aufgetretenen intrahepatischen Herden als Rezidiv, die zentral liegen und einer Re-Resektion nicht zugänglich sind. Der Stellenwert der intraoperativen Radiofrequenzablation zusätzlich zu einer Oxaliplatin-basierten syste- 
mischen Chemotherapie wird gegenwärtig in einer multizentrischen randomisierten Studie überprüft (CLOCC Trial, EORTC 40004).

Günther/Tacke: Eine Erfolg versprechende Kombination beider Verfahren ist gegeben, wenn unterschiedlich große Tumoren in beiden Leberlappen vorhanden sind, etwa im Sinne einer Resektion des Leberlappens mit der größeren Tumorlast und einer perkutanen Ablation in dem verbleibenden Leberlappen. Dies wurde schon mehrfach mit einer interventionellradiologischen Portalvenenembolisation zur Hypertrophie des verbleibenden Leberlappens kombiniert.

Die Kriterien zur intraoperativen Thermoablation wurden bereits unter Punkt 8 erläutert. Wenn die Läsionen auch intraoperativ unter Ultraschallkontrolle nicht sicher zu abladieren sind, kann nach operativer Resektion einzelner Herde eine Indikation zu CT- oder MRT-gesteuerten Ablation dieser Herde bestehen, wenn mit diesen Verfahren ein sicherer $\mathrm{Zu}$ gangsweg gewährleistet wird. Allerdings sollte in diesen Fällen die perkutane Ablation der Operation vorangestellt werden, da die Möglichkeiten der artefaktfreien Bildgebung und Interventionssteuerung beim nichtoperierten Patienten besser sind und operationsbedingte Leberveränderungen entfallen.

Helmberger: Die Frage sollte anders gestellt werden: Warum soll eine Resektion mit einer Thermoablation kombiniert werden? Soll so ein ursprünglich nichtresektabler Patient resektabel gemacht werden? Das würde aber bedeuten, dass dann das abladierte Areal automatisch als R0-abladiert gilt. Hierzu fehlen aber bisher noch Studiendaten. Wird andererseits das Ablationssystem als erweitertes chirurgisches Instrumentarium aufgefasst und werden die üblichen chirurgischen Resektionskriterien eingehalten, so ist der Gedanke der minimal invasiven Tumortherapie ad absurdum geführt. Ketzerisch könnte man sogar fragen: «Wenn ein Herd unter diesen Umständen perkutan behandelbar ist, warum denn dann überhaupt OP?»

Die postoperative Thermoablation ist sicherlich dann indiziert, wenn nach vorangegangener Resektion technisch oder aus onkologischen Gründen eine Re-Resektion nicht mehr in Frage kommt, und es sinnvoll erscheint, einen eventuell neuen oder verbliebenen Herd zu abladieren. Dieses Konzept erschient mir grundsätzlich aufgrund der Monitoring-Probleme auch für das kombinierte intraoperative Vorgehen anwendbar, d.h., ich halte es für sicherer einen Herd, der intraoperativ abladiert werden soll, da er für eine Resektion ungeeignet ist (in der Regel lagebedingt), eher perkutan zu abladieren, da dabei Herd und Ablationsvorgang besser kontrolliert werden können, als wenn nur Ultraschall zur Verfügung steht.
Freund: Eine Kombination von Resektion und intraoperativer Thermoablation wird als vorteilhaft berichtet. Allerdings handelt es sich um retrospektive Auswertungen, die Selektion ist unklar. Eine eindeutige Datenlage ist nicht vorhanden.

\section{Frage 12: Bei welcher Metastasenart ist eine Leber- transplantation onkologisch sinnvoll?}

Bechstein: Eine Lebertransplantation erscheint aufgrund der vorliegenden Daten in Einzelfällen von Metastasen neuroendokriner Tumoren onkologisch sinnvoll. Allerdings gilt dies eher für jüngere Patienten und eher für Patienten, bei denen die Metastasen metachron aufgetreten sind, und immer dann, wenn die Transplantation nicht als Multiviszeraltransplantation durchgeführt wird.

Freund: Am besten etabliert ist die Lebertransplantation beim HCC [30, 31], doch das trifft die Frage nicht wirklich. Bei Lebermetastasen neuroendokriner Tumoren sind gute Ergebnisse für die Lebertransplantation in einer kleinen Serie berichtet worden [32]. Berichte zum Mammakarzinom oder kolorektalem Karzinom sind anekdotisch und können nicht wirklich zu Schlüssen führen [33, 34].

\section{Teilnehmer}

Prof. Dr. med. Wolf Otto Bechstein

Klinik für Allgemein- und Gefäßchirurgie, Universitätsklinikum

Johann-Wolfgang Goethe-Universität Frankfurt am Main

Theodor-Stern-Kai 7, D-60590 Frankfurt

E-mail wolf.bechstein@kgu.de

Prof. Dr. med. Rolf Günther

Klinik für Radiologische Diagnostik, RWTH Aachen

Pauwelsstraße 30, D-52074 Aachen

E-mail radiologie@ukaachen.de

PD Dr. med. Josef Tacke

Klinik für Radiologische Diagnostik, RWTH Aachen

Pauwelsstraße 30, D-52074 Aachen

E-mail tacke@rad.rwth-aachen.de

PD Dr. T. Helmberger

Institut für Klinische Radiologie

Klinikum Großhadern, Universität München

Marchioninistr. 15, D-81377 München

E-mail Thomas.Helmberger@ikra.med.uni-muenchen.de

Prof. Dr. Mathias Freund

Abteilung Hämatologie und Onkologie

Klinik und Poliklinik für Innere Medizin, Universität Rostock

Ernst-Heydemann-Straße 6, D-18055 Rostock

Tel. 0381494 74-20, Fax -22

E-mail mathias.freund@med.uni-rostock.de 
1 Kinkel K, Lu Y, Both M, Warren RS, Thoeni RF: Detection of hepatic metastases from cancers of the gastrointestinal tract by using noninvasive imaging methods (US, CT, MR imaging, PET): A meta-analysis. Radiology 2002;224:748-756.

2 Esteban JM, Molla MA, Tomas C, Maldonado L: Improved detection of liver metastases with contrast-enhanced wideband harmonic imaging: Comparison with CT findings. Eur J Ultrasound 2002; 15:119-126.

3 Vogl TJ, Schwarz W, Blume S, Pietsch M, Shamsi K, Franz M, et al: Preoperative evaluation of malignant liver tumors: Comparison of unenhanced and SPIO (Resovist)-enhanced MR imaging with biphasic CTAP and intraoperative US. Eur Radiol 2003;13:262-272.

4 Giacchetti S, Itzhaki M, Gruia G, Adam R, Zidani R, Kunstlinger F, Brienza S, Alafaci E, BertheaultCvitkovic F, Jasmin C, Reynes M, Bismuth H, Misset JL, Levi F: Long-term survival of patients with unresectable colorectal cancer liver metastases following infusional chemotherapy with 5-fluorouracil, leucovorin, oxaliplatin and surgery. Ann Oncol 1999;10:663-669. Comment in Ann Oncol 2000;11:243-244.

5 Tanaka K, Adam R, Shimada H, Azoulay D, Levi F, Bismuth H: Role of neoadjuvant chemotherapy in the treatment of multiple colorectal metastases to the liver. Br J Surg 2003;90:963-969.

6 Adam R, Avisar E, Ariche A, Giachetti S, Azoulay D, Castaing D, et al: Five-year survival following hepatic resection after neoadjuvant therapy for nonresectable colorectal. Ann Surg Oncol 2001;8: 347-353.

7 Bismuth H, Adam R, Levi F, Farabos C, Waechter F, Castaing D, et al: Resection of nonresectable liver metastases from colorectal cancer after neoadjuvant chemotherapy. Ann Surg 1996;224:509-520.

8 Giacchetti S, Itzhaki M, Gruia G, Adam R, Zidani $\mathrm{R}$, Kunstlinger F, et al: Long-term survival of patients with unresectable colorectal cancer liver metastases following infusional chemotherapy with 5-fluorouracil, leucovorin, oxaliplatin and surgery. Ann Oncol 1999;10:663-669.

9 EORTC GITCCG. Protocol 40983: Pre- and PostOperative Chemotherapy with Oxaliplatin 5FU/LV versus Surgery alone in Resectable Liver Metastases from Colorectal Origin - Phase III Study. 2003.

10 Lorenz M, Muller HH, Schramm H, Gassel HJ, Rau HG, Ridwelski K, et al: Randomized trial of surgery versus surgery followed by adjuvant hepatic arterial infusion with 5-fluorouracil and folinic acid for liver metastases of colorectal cancer. German Cooperative on Liver Metastases (Arbeitsgruppe Lebermetastasen). Ann Surg 1998;228:756762.
11 Kemeny MM, Adak S, Gray B, Macdonald JS, Smith T, Lipsitz S, et al: Combined-modality treatment for resectable metastatic colorectal carcinoma to the liver: Surgical resection of hepatic metastases in combination with continuous infusion of chemotherapy - an intergroup study. J Clin Oncol 2002;20:1499-1505.

12 Kemeny N, Huang Y, Cohen AM, Shi W, Conti JA, Brennan MF, et al: Hepatic arterial infusion of chemotherapy after resection of hepatic metastases from colorectal cancer. N Engl J Med 1999;341: 2039-2048.

13 Kerr DJ, McArdle CS, Ledermann J, Taylor I, Sherlock DJ, Schlag PM, Buckels J, Mayer D, Cain D, Stephens RJ; Medical Research Council's colorectal cancer study group, for the European Organisation for Research and Treatment of Cancer Colorectal Cancer Study Group: Intrahepatic arterial versus intravenous fluorouracil and folinic acid for colorectal cancer liver metastases: A multicentre randomised trial. Lancet 2003;361:368-373. Comment in Lancet 2003 ;361:358-359; Lancet 2003;361: 1742-1743; author reply 1743.

14 Heinrich S, Petrowsky H, Schwinnen I, Staib-Sebler E, Gog C, El Ganainy A, et al: Technical complications of continuous intra-arterial chemotherapy with 5-fluorodeoxyuridine and 5-fluorouracil for colorectal liver metastases. Surgery 2003;133:40-48.

15 Stangl R, Altendorf-Hofman A, Charnley RM, Scheele J: Factors influencing the natural history of colorectal liver metastases. Lancet 1994;343:14051410.

16 Fong Y, Cohen AM, Fortner JG, Enker WE, Turnbull AD, Coit DG, et al: Liver resection for colorectal metastases. J Clin Oncol 1997;15:938-946.

17 Pinson CW, Wright JK, Chapman WC, Garrard CL, Blair TK, Sawyers JL: Repeat hepatic surgery for colorectal cancer metastasis to the liver. Ann Surg 1996;223:765-773.

18 Vogl TJ, Mack MG, Balzer JO, Engelmann K, Straub R, Eichler K, et al: Liver metastases: Neoadjuvant downsizing with transarterial chemoembolization before laser-induced thermotherapy. Radiology 2003;229:457-464.

19 Mutsaerts EL, Van Coevorden F, Krause R, Borel R, I, Strobbe LJ, Prevoo W, et al: Initial experience with radiofrequency ablation for hepatic tumours in the Netherlands. Eur J Surg Oncol 2003;29:731734.

20 Giovannini M, Moutardier V, Danisi C, Bories E, Pesenti C, Delpero JR: Treatment of hepatocellular carcinoma using percutaneous radiofrequency thermoablation: Results and outcomes in 56 patients. $\mathrm{J}$ Gastrointest Surg 2003;7:791-796.

21 Stella M, Minuto MN, Pasqualini M, Percivale A, Profeti A, Serafini G, et al: Intraoperative use of radiofrequency thermoablation of liver tumors: Considerations on indications and related therapeutic aspects (in Italic). Ann Ital Chir 2002;73: 511-516.
22 Hansler J, Witte A, Strobel D, Wein A, Bernatik T, Pavel M, et al: Radio-frequency-ablation (RFA) with wet electrodes in the treatment of primary and secondary liver tumours (in German). Ultraschall Med 2003;24:27-33.

23 Elias D, Goharin A, El Otmany A, Taieb J, Duvillard P, Lasser P, et al: Usefulness of intraoperative radiofrequency thermoablation of liver tumours associated or not with hepatectomy. Eur J Surg Oncol 2000;26:763-769.

24 Vogl TJ, Mack MG, Roggan A: Magnetresonanztomographisch gesteuerte laserinduzierte Thermotherapie von Lebermetastasen. Dtsch Ärztebl 2000; 97:B-2039-2044.

25 Carlini M, Lonardo MT, Carboni F, Petric M, Vitucci C, Santoro R, et al: Liver metastases from breast cancer. Results of surgical resection. Hepatogastroenterology 2002;49:1597-1601.

26 Elias D, Cavalcanti DA, Eggenspieler P, Plaud B, Ducreux M, Spielmann M, et al: Resection of liver metastases from a noncolorectal primary: Indications and results based on 147 monocentric patients. J Am Coll Surg 1998;187:487-493.

27 Schmidbauer S, Ladurner R, Juckstock H, Trupka AW, Mussack T, Hallfeldt KK: Surgical and adjuvant therapy of neuroendocrine tumors of the gastrointestinal tract and their metastases. A retrospective analysis of personal patient group (in German). Chirurg 2001;72:945-952.

28 Ahlman H, Westberg G, Wangberg B, Nilsson O, Tylen U, Schersten T, et al: Treatment of liver metastases of carcinoid tumors. World J Surg 1996;20: 196-202.

29 Lehnert T, Knaebel HP: Diagnosis and therapy of liver metastases of neuroendocrine tumors (in German). Chirurg 1997;68:122-131.

30 Khakhar A, Solano E, Stell D, Bloch M, Dale C, Burns P, et al: Survival after liver transplantation for hepatocellular carcinoma. Transpl Proc 2003;35: 2438-2441.

31 Adam R, McMaster P, O'Grady JG, Castaing D, Klempnauer JL, Jamieson N, et al: Evolution of liver transplantation in Europe: Report of the European Liver Transplant Registry. Liver Transpl 2003;9:1231-1243.

32 Cahlin C, Friman S, Ahlman H, Backman L, Mjornstedt L, Lindner P, et al: Liver transplantation for metastatic neuroendocrine tumor disease. Transpl Proc 2003;35:809-810.

33 Honore C, Detry O, De Roover A, Meurisse M, Honore P: Liver transplantation for metastatic colon adenocarcinoma: Report of a case with 10 years of follow-up without recurrence. Transpl Int 2003;16:692-693.

34 Wilson JM, Carder P, Downey S, Davies MH, Wyatt JI, Brennan TG: Treatment of metastatic breast cancer with liver transplantation. Breast J 2003;9:126-128. 